\title{
Confronting the COVID-19 pandemic: A view from the laboratory
}

\author{
Gary Procop, MD, MS ${ }^{1}$
}

Author affiliations are listed at the end of this article.

A retrospective review of salient features of the early-to-mid phases of the COVID-19 pandemic is provided from the perspective of the clinical laboratory. Lessons learned and possible improvements are opined. These include the importance of maintaining a robust laboratory infrastructure, increased public health funding, the partnership between public health and hospital/commercial laboratories, and the critical importance of sound, scientific assessment, even during a pandemic crisis.

Correspondence to: Gary W. Procop, MD, MS Cleveland Clinic Lerner College of Medicine procopg@ccf.org

\section{KEYWORDS}

COVID-19, SARS-CoV-2, Laboratory, Testing

\section{AND SO, IT BEGINS...}

The COVID-19 pandemic, which hit North America like a firestorm, began on January 21st when the first patient with SARS-CoV-2 was confirmed, and it continues to rage today ${ }^{[1]}$. The public health sector was immediately overwhelmed, although they initially intended to provide testing for potentially infected individuals. At the time, clinical laboratories were not permitted by FDA mandate to perform testing for SARS-CoV-2. This was particularly frustrating for high-complexity laboratories that already perform a wide variety of laboratory-developed molecular assays for infectious agents ${ }^{[2]}$. This changed by February 29th, when it became apparent that there was no possibility that the public health system could handle the impending volume of SARS-CoV-2 tests. At this point, the FDA opened a pathway for medical laboratories to offer testing, albeit through a cumbersome emergency use authorization (EUA) process. This compounded frustrations for laboratorians, since the type of testing (i.e. reverse transcription-polymerase chain reaction [RT-PCR]), is commonly used by high-complexity molecular microbiology laboratories for a variety of pathogens. Otherwise stated, the opportunity to provide local testing for patients was becoming available, but at the price of substantial bureaucratic paperwork in the midst of a crisis. The early timeline through April 5th, 2020, with steps and missteps, has been outlined by the Washington Post ${ }^{[1]}$.

\section{Lessons:}

1. The public health systems in the U.S. (i.e., federal, state, and county) are woefully underfunded and understaffed, and, therefore, unprepared to respond to a global respiratory viral pandemic.

2. The CDC is optimally positioned to develop and validate tests for novel pathogens, and then transparently provide the materials and/or methods for use by accredited private sector laboratory partners.

3. The imposition of new and inconsistent (i.e., changing) regulations hinder testing and delay patient care.

Considerations: The nation witnessed significant backlogs of testing by the largest, most efficient commercial reference laboratories in the country. When test results for a communicable agent are significantly delayed, the results become clinically meaningless, apart from documenting the cause of an event. It highly unlikely that stable and sufficient funding for 
public health will be forthcoming to the extent to enable the testing required to address a respiratory viral pandemic. The CDC is uniquely positioned with dedicated professional medical scientists and laboratory facilities to develop and test assays that could then be made freely available to hospital systems with laboratories that are capable of performing high-complexity molecular diagnostic testing. Partnerships between public and private laboratories have a greater likelihood of success than either of these entities acting alone.

Laboratories that are currently accredited under U.S. law to produce and perform laboratory-developed tests in high-complexity molecular laboratories should be allowed to, regardless of the pathogen targeted, without the imposition of additional regulations and burdensome submissions to the FDA. Highly-accurate tests for emerging pathogens could thereby be more rapidly offered in the clinical setting, with the same safety and other regulatory oversights that currently exist for other highly-complex tests.

\section{MAINTAIN AN ADEQUATE LABORATORY INFRA- STRUCTURE}

Extensive outsourcing of pathology and laboratory medicine services, which may look good on paper as an administrative cost-saving initiative, significantly weakens the infrastructure of healthcare facilities and diminishes the collaboration between clinicians and their colleagues in laboratory medicine. The latter collaboration brings an in-depth understanding of pathologic findings and laboratory studies to patient care that complements the knowledge and skills of patient-facing healthcare providers for optimal care delivery. It is not possible for a laboratory weakened by outsourcing and lack of investment to adequately respond to a pandemic. Such a laboratory is dead in the water in a pandemic and completely reliant on outsourced testing, which may take two weeks or more for test results to be returned ${ }^{[3]}$. The Cleveland Clinic was able to offer the first SARSCoV-2 test in Ohio because of a recognition of the value of pathology and laboratory medicine to world-class care, and fiscally-responsible investments made over time in highly-skilled personnel, space, and instrumentation ${ }^{[4]}$.
Lessons:

1. Investing in the infrastructure of the local laboratory helps serve patients and providers alike by providing timely, clinically meaningful results, without the delays associated with send-out testing, which is particularly important in a pandemic.

2. Pathologists and laboratory medicine practitioners are important members of the healthcare team, and their skills, which extend beyond the laboratory, should be employed to the fullest to optimize care delivery.

\section{TO ACT AS A UNIT: THE LABORATORY IS PART OF THE TEAM}

The central role of accurate and timely testing in healthcare delivery is known, but never before has testing and laboratory medicine played a more visible role in healthcare delivery. Are there enough tests? Why are there delays in testing? Who should be tested? Does serology play a role in diagnostic testing? How do tests compare? Which test should I use in which setting? What is the difference between diagnostic tests versus screening tests? Is this test reliable? All of these questions and more have been asked numerous times throughout the pandemic, and laboratory professionals in partnership with their clinical colleagues have worked to answer them in an evidence-based manner.

Although our laboratory was able to validate and offer the CDC 2019 nCoV Real-Time RT-PCR Diagnostic Panel, there were significant limitations on the number of tests that could be performed due to supply constraint ${ }^{[4,5]}$. If this information was not communicated to health system leadership teams, and decisions were not made concerning testing triage, then testing resources would be exhausted on a first-come, first-served basis. This would translate into many "worried well" being tested, while other patients more in need of a test went without. Additionally, it was critical to have the ability to compare test performance characteristics so that the optimal test could be paired with the appropriate patient care setting (e.g., inpatient versus ambulatory $)^{[6]}$.

The Incident Command structure and process at our institution, which was initially assembled to prepare 
for the pandemic, continued on an ongoing basis for many months into the pandemic. In this setting, representatives of all vested parties met regularly to understand the challenges and limitations we were confronting and likely would confront (e.g., impending supply shortages), as a health system. Meetings were held twice per day early in the pandemic, then changed to daily upon more stabilization. This proved an excellent model for shared decision-making, which has been demonstrated by others ${ }^{[7-9]}$. The Laboratory Stewardship committee has continued to meet in this shared stewardship model, after the suspension of the standing Incident Command meetings, to continue to address challenges associated with testing.

\section{NEW APPROACHES}

The methods that are used in laboratory medicine have evolved and been refined over centuries. It has been unsettling to watch how quickly some will abandon tried-and-true approaches in a scrambled effort to provide a service (i.e., testing) due to limited supplies. Necessity is indeed the mother of invention, but adherence to sound scientific principles is critical for the assessment of new approaches for optimal patient care and safety.

The abandonment of optimal methods that are used to provide a "gold standard" test result (e.g. nucleic acid extraction followed by RT-PCR) usually comes at a price. Most commonly, with respect to COVID-19 testing, some analytical sensitivity is lost at the expense of speed, ease-of-use, and/or cost reduction. The impact of this loss of sensitivity may be entirely acceptable but should be clearly understood and disclosed to the provider so as to inform the best patient care decisions. Three significant changes in testing for SARS-CoV-2 testing have been explored, including the use of saliva as an alternate specimen type, the pooling of clinical specimens, and the FDA EUA approval of at-home testing, which includes antigen testing rather than nucleic acid amplification.

The shortage of nasopharyngeal swabs early in the pandemic necessitated the use of nasal midturbinate and more proximal nasal swabbing as alternatives ${ }^{[10]}$. Saliva has also been explored as an alternate diagnostic specimen, with quality reported from superior to inferior compared to nasopharyngeal swabbing ${ }^{[11-14]}$. Our evaluation demonstrated the essential equivalence of saliva for the diagnosis of symptomatic patients, but saliva specimens had a lower viral load compared with nasopharyngeal swab specimens ${ }^{[15]}$.

The shortage of testing reagents and other supplies has forced the consideration of pooling clinical specimens, which is a technique not used in the clinical laboratory ${ }^{[16]}$. Pooling allows the testing of multiple patients with one test at the expense of a slightly lowered sensitivity. FDA guidance requires that pool testing have an $85 \%$ positive percent agreement (PPA) compared with neat or non-diluted testing of clinical specimens. Our evaluation of a commonly used platform demonstrated that 10:1 would meet the $85 \%$ PPA and that only specimens with low viral loads would be missed ${ }^{[17]}$. Providers need to determine if this is acceptable for the populations being tested.

Three home tests for COVID-19 have been recently released. One of these (i.e. Lucira ${ }^{\text {TM }}$ COVID-19 All-In-One Test Kit [Lucira Health, Emeryville, CA]) is an isothermal amplification assay, whereas the other two (i.e. Ellume COVID-19 Home Test [Ellume, East Brisbane, Australia] and BinaxNOW COVID-19 Ag Card Home Test [Abbott, Abbott Park, IL]) are antigen detection assays. These assays, although slightly less sensitive than laboratory-based molecular tests, offer several advantages. These tests are relatively inexpensive, easy to use and provide results in $\mathbf{3 0}$ minutes or less. Additional advantages include the maintenance of isolation (i.e. patients do not need to travel for testing) and the preservation of personal protective equipment since healthcare providers do not need to take the specimen. Abundant performance information is available on company websites, including FDA EUA submission data, as well as a review in a pending publication of the Cleveland Clinic Journal of Medicine. The advantages and limitations of antigen tests have been reviewed ${ }^{[18]}$. Providers should review the strengths and limitations of these assays to determine if they fit the needs of their patients. 


\section{CONCLUSION}

The initial focus of the response to the pandemic from the laboratory was offering high-quality, reliable tests for a virus none of us had ever encountered; however, this was only feasible in laboratories with a sound infrastructure. An investment in your laboratory and partnerships with laboratory-based care providers will pay dividends beyond the few dollars saved by outsourcing tests. The partnerships between pathologists, laboratorians, and clinical providers were critical for triage needed for limited resources. The sound principles of medical-scientific assessment and test validation/verification must be adhered to, even in times of crisis, to deliver the best, most reliable tests for optimal patient care.

\section{AUTHOR AFFILIATIONS}

1. Medical Director, Molecular Microbiology, Mycology, Parsitology \& Virology Laboratories, The Belinda Yen-Lieberman, PhD \& James M. Liberman, MD Endowed Chair in Clinical Microbiology, Vice Chair Pathology Education, Pathology \& Laboratory Medicine Institute, Program Director, Clinical Microbiology Fellowship, Professor of Pathology, Cleveland Clinic Lerner College of Medicine, Cleveland Clinic

\section{REFERENCES}

1. What we know about delays in coronavirus testing, in The Washington Post. 2020: Washington, D.C.

2. Patel, R., et al. Amid COVID-19, ASM Voices Concerns About Clinical Access to Tests. 2020 [cited 2021 1/10/2021]; Available from: https://asm.org/ Articles/Policy/2020/Amid-COVID-19,-ASM-Advocates-for-Changes-to-FDA-Em.

3. Paul, D., I. Lovett, and D. Frosch, Why a Coronavirus Test Result Takes Two Weeks or More in The Wall Street Journal. 2020.

4. Reddy, A.J., et al., Operationalizing COVID-19 testing: Who, what, when, where, why, and how. Cleve Clin J Med, 2020.

5. Lu, X., et al., US CDC Real-Time Reverse Transcription PCR Panel for Detection of Severe Acute Respiratory Syndrome Coronavirus 2. Emerg Infect
Dis, 2020. 26(8).

6. Procop, G.W., et al., A Comparison of Five SARSCoV-2 Molecular Assays With Clinical Correlations. Am J Clin Pathol, 2020.

7. Cook, J., Incident Command in the Time of COVID-19. Lab Med, 2020. 51(6): p. e78-e82.

8. Desai, S., et al., Rapid-Cycle Improvement During the COVID-19 Pandemic: Using Safety Reports to Inform Incident Command. Jt Comm J Qual Patient Saf, 2020. 46(12): p. 715-718.

9. Tosh, P.K., et al., Elements of an Effective Incident Command Center. Mayo Clin Proc, 2020. 95(9S): p. S3-S7.

10. Tu, Y.P., et al., Swabs Collected by Patients or Health Care Workers for SARS-CoV-2 Testing. N Engl J Med, 2020. 383(5): p. 494-496.

11. Chen, J.H., et al., Evaluating the use of posterior oropharyngeal saliva in a point-of-care assay for the detection of SARS-CoV-2. Emerg Microbes Infect, 2020. 9(1): p. 1356-1359.

12. Leung, E.C., et al., Deep throat saliva as an alternative diagnostic specimen type for the detection of SARS-CoV-2. J Med Virol, 2020.

13. Pasomsub, E., et al., Saliva sample as a non-invasive specimen for the diagnosis of coronavirus disease 2019: a cross-sectional study. Clin Microbiol Infect, 2020.

14. Wyllie, A.L., et al., Saliva or Nasopharyngeal Swab Specimens for Detection of SARS-CoV-2. N Engl J Med, 2020.

15. Procop, G.W., et al., A Direct Comparison of Enhanced Saliva to Nasopharyngeal Swab for the Detection of SARS-CoV-2 in Symptomatic Patients. J Clin Microbiol, 2020. 58(11).

16. Rohde, R. COVID-19 Pool Testing: Is It Time to Jump In? 2020 [cited 2020 10/4/2020]; Available from https://asm.org/Articles/2020/July/COVID19-Pool-Testing-Is-It-Time-to-Jump-In.

17. Procop, G.W., et al., Asymptomatic Patient Testing After 10:1 Pooling Using the Xpert Xpress SARSCoV-2 Assay. Am J Clin Pathol, 2021.

18. Procop, G.W., On Antigen Testing. 2020: ASCP News. p. https://www.ascp.org/content/news-archive/news-detail/2020/11/11/on-antigen-testing-for-covid-19. 